\title{
Modeling of the Experimental Molecular-Based Ring-Shaped Nanomagnets
}

\author{
M. Antkowiak, P. KozŁowski, G. Musią, W. Florek, G. Kamieniarz*
} Faculty of Physics, A. Mickiewicz University, Umultowska 85, 61-614 Poznań, Poland

AND F. EsPosito

Dipartimento di Scienze Fisiche, Universita di Napoli, Napoli, Italy

Quantum transfer matrix technique and numerically exact diagonalization method are applied to the Heisenberg spin systems to model ring-shaped molecules. Two cases are investigated: (i) a dozen of $S=1$ spins with additional biquadratic exchange and (ii) a dimetallic molecule $\mathrm{Cr}_{7} \mathrm{Cd}$, where it is assumed that exchange anisotropy is determined in a local coordination system. In the latter case the calculated susceptibility is compared with experimental results.

PACS numbers: 75.40.Cx, 75.40.Mg

\section{Introduction}

Molecular-based nanomagnets are important in fundamental physics and are good candidates for applications in the domain of magnetic storage, molecular spintronics and quantum computing $[1,2]$. Relatively not so large dimensions of eigenproblems make possible to apply exact (at least numerically) methods to quite complicated models $[3,4]$. In this communication we present two model Hamiltonians with results obtained by two different methods.

At first we study influence of biquadratic exchange on the ground state energy and ordering of excited levels in the case of a dodecanuclear ring of spins $S=1$ in the presence of single-ion anisotropy. Hence, the model Hamiltonian is as follows:

$$
\mathcal{H}_{1}=-\sum_{i=1}^{12} J \boldsymbol{S}_{i} \cdot \boldsymbol{S}_{i+1}+K\left(\boldsymbol{S}_{i} \cdot \boldsymbol{S}_{i+1}\right)^{2}-D\left(\boldsymbol{S}_{i}^{z}\right)^{2}
$$

The second non-perturbative method applied is based on the so-called quantum transfer matrix (QTM) [3, 4]. In this paper this technique is used to calculate the magnetic susceptibility of the $\mathrm{Cr}_{7} \mathrm{Cd}$ dimetallic ring $(S=3 / 2$ for chromium ions, $\mathrm{Cd}$ is a non-magnetic substitute) $[5,6]$. We use the anisotropic Hamiltonian introduced by Piligkos et al. [7]:

$$
\begin{aligned}
\mathcal{H}_{2} & =\sum_{i=1}^{6}\left(J \boldsymbol{S}_{i} \cdot \boldsymbol{S}_{i+1}+\boldsymbol{S}_{i} R D_{l} R^{-1} \boldsymbol{S}_{i+1}\right) \\
& +\sum_{i=1}^{7}\left[D\left(S_{i}^{z}\right)^{2}-g \mu_{\mathrm{B}} B\left(S_{i}^{z} \cos \theta+S_{i}^{x} \sin \theta\right)\right],
\end{aligned}
$$

where $\theta$ is the angle between the magnetic field $B$ and the $z$ axis, $D_{l}$ is a diagonal matrix $\left(D_{l}^{x x}=D_{l}^{y y}=-D_{l}^{z z} / 2=\right.$ $-d$ ) describing exchange anisotropy in local coordination system and $R$ is a proper rotation matrix [7].

\section{Results}

Methods described in the previous paper [4] have been modified to include the additional biquadratic term. We

\footnotetext{
* corresponding author; e-mail: gjk@amu.edu.pl
}

have calculated the basis of orthonormal states classified according to the total spin $S$, the magnetization $M$ and a label $k$ with $0 \leq|k| \leq 6$ playing the role of a wave number [4]. For $k=0,6$ an additional label \pm , describing symmetry under the reflection $j \leftrightarrow(13-j)$, can be introduced. In this basis, for the moderate values of the parameter $D$, the mean values of $\boldsymbol{S}^{2}$ are very close to the exact eigenvalues, so that we can still group the energy levels as the multiplets of the (approximate) total spin and states in a given $S$-multiplet will be denoted by a pair $(M, k \pm)$ or $(M,|k|)$ for $0<|k|<6$.

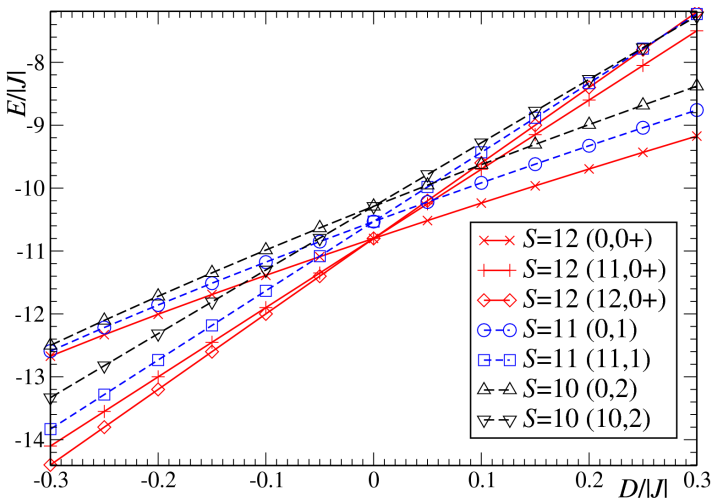

Fig. 1. Energy levels of the states $S=10,11,12$ as a function of the anisotropy $D$ for $K=0.1$ and $J=1$.

At first we have analysed the ordering of the lowest lying energy levels for $J=1$ and $K=0.1$ (see Fig. 1). In the isotropic limit $(D=0)$ the non-zero biquadratic exchange modifies this order: the second excited state has $S=10$, whereas for $K=0$ three consecutive excited levels have $S=11$ with $k=1,2,3[3]$.

The field-induced changes of the ground state have been analysed for antiferromagnetic exchange $(J=-1)$. The degeneracy of each $S$-multiplet is lifted by an external magnetic field $B>0$ due to the Zeeman term $g \mu_{\mathrm{B}} B M$ : the lowest lying state is the singlet with $M=-S$. As the external field is increased from $B=0$, the ground state switches to $S=1,2, \ldots, 12$ for "criti- 


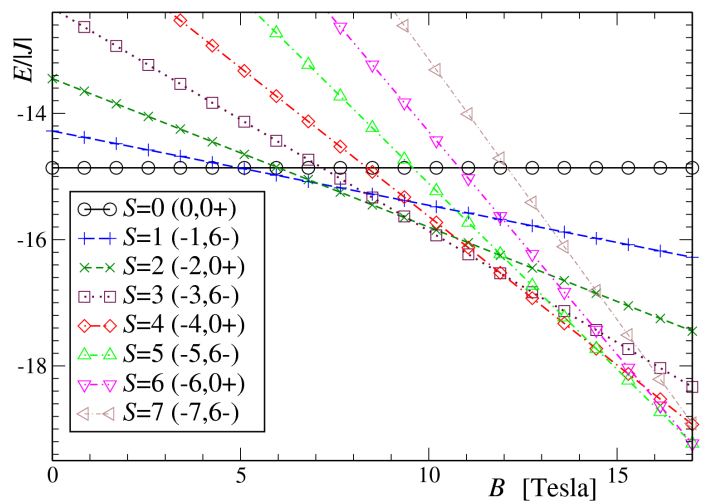

Fig. 2. The field-dependence of the lowest-lying states for $J=-1, D=-0.1$, and $K=0.1$.

cal" values of $B_{S}$. For $B=0$ the non-zero anisotropy $D$ yields splitting of $S$-multiplets into $S+1$ levels, so the "starting points" for each level and energy gaps between them are changed. The energies of lowest lying states for $0 \geq M \geq-7$ have been plotted for $D=-0.1$ and $K=0.1$ (see Fig. 2). Except for the first critical value $B_{1} \approx 4.96 \mathrm{~T}$ the crossings are observed when $B$ is increased by approximately $2.4 \mathrm{~T}$ : the next shown critical values are shifted by $2.10,2.42,2.46,2.48$ and $2.54 \mathrm{~T}$. It means that the first energy gap is larger than predicted by the Landé interval rule [4].

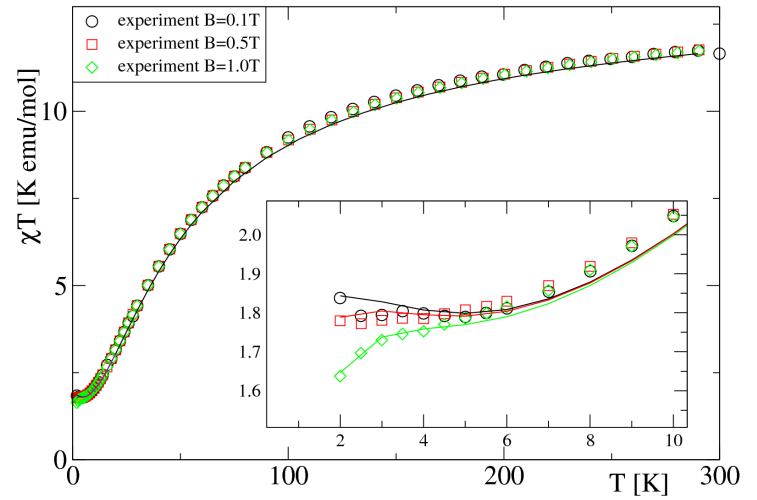

Fig. 3. Experimental (points) and numerical (lines) values of $\chi T$ for $\mathrm{Cr}_{7} \mathrm{Cd}$. In the inset low-temperature regime/region.

Studying the model for $\mathrm{Cr}_{7} \mathrm{Cd}$ molecule by the QTM technique we have taken the parameters obtained by fitting to the EPR-spectra by Pilikgos et al. [7]: $J=16.604 \mathrm{~K}, d=-0.1525 \mathrm{~K}, D=-0.1928 \mathrm{~K}$, $g=1.98$. Magnetic susceptibility data were recorded in the $2-300 \mathrm{~K}$ temperature range in applied magnetic fields of $0.1,0.5$ and $1 \mathrm{~T}$ (perpendicular to the direction of the measurement) [6]. The diamagnetic correction 0.001 has been added to the numerical results. It can be clearly seen that we have obtained good agreement with experimental results (see Fig. 3). We would like to put stress for the results below $6 \mathrm{~K}$, where distinct field-dependent behaviour of $\chi$ is observed: our numerical results confirm smaller values of susceptibility for higher magnetic field and, moreover, very well fit to the experimental results for $B=1 \mathrm{~T}$ below $3 \mathrm{~K}$, when $\chi T$ decreases almost linearly.

\section{Conclusions}

This result shows the relevance of the anisotropy effects in determination of the thermodynamic and magnetic properties of molecular magnets. We have shown that quite complicated models, based on the Heisenberg Hamiltonian, of such molecules can be solved exactly and one can fit the microscopic parameters with high precision. It should be underlined that additional terms in the model Hamiltonians can be taken into account almost at no extra computational cost. Moreover, in the QTM method no energy levels are neglected which leads to reliable results both in low and high temperatures.

\section{Acknowledgments}

This work was supported in part by the MNiSW within the project No. N N202 230137. Numerical calculations were carried out on the platforms of the Supercomputing and Networking Center in Poznań.

\section{References}

[1] M. Mannini, F. Pineider, Ph. Sainctavit, Ch. Danieli, E. Otero, C. Sciancalepore, A.M. Talarico, M.-A. Arrio, A. Cornia, D. Gatteschi, R. Sessoli, Nature Mater. 8, 194 (2009).

[2] G.A. Timco, S. Carretta, F. Troiani, F. Tuna, R.J. Pritchard, Ch.A. Muryn, E.J.L. McInnes, A. Ghirri, A. Candini, P. Santini, G. Amoretti, M. Affronte, R.E.P. Winpenny, Nature Nanotechnol. 4, 173 (2009).

[3] G. Kamieniarz, M. Haglauer, G. Musiał, A. Caramico D'Auria, F. Esposito, D. Gatteschi, Inorg. Chim. Acta 360, 3941 (2007).

[4] G. Kamieniarz, P. Kozłowski, G. Musiał, W. Florek, M. Antkowiak, M. Haglauer, A. Caramico D'Auria, F. Esposito, Inorg. Chim. Acta 361, 3690 (2008).

[5] E.J.L. McInnes, S. Piligkos, G.A. Timco, R.E.P. Winpenny, Coord. Chem. Rev. 249, 2577 (2005).

[6] P. Kozłowski, G. Kamieniarz, M. Antkowiak, F. Tuna, G.A. Timco, R.E.P. Winpenny, Polyhedron 28, 1852 (2009).

[7] S. Piligkos, H. Weihe, E. Bill, F. Neese, H. el Mkami, G.M. Smith, D. Collison, G. Rajaraman, G.A. Timco, R.E.P. Winpenny, E.J.L. McInnes, Chem. Eur. J. 15, 3152 (2009). 